\title{
Outcome of Life-Threatening Arrhythmias Among Patients Presenting in an Emergency Setting at a Tertiary Hospital in Accra-Ghana
}

\author{
Alfred Doku ( $\square$ dokukavin@gmail.com ) \\ University of Ghana Medical School, Korle-Bu Teaching Hospital \\ Bernard Yeboah-Asiamah Asare \\ Curtin University \\ Richard Osei \\ National Cardiothoracic Centre, Korle-Bu Teaching Hospital \\ Christian Owoo \\ University of Ghana Medical School, Korle-Bu Teaching Hospital \\ Robert Djagbletey \\ University of Ghana Medical School, Korle-Bu Teaching Hospital \\ Joseph Akamah \\ University of Ghana Medical School, Korle-Bu Teaching Hospital \\ Ernest Aniteye \\ University of Ghana Medical School, Korle-Bu Teaching Hospital \\ Dzifa Ahadzi \\ Korle-Bu Teaching Hospital
}

\section{Research Article}

Keywords: Arrhythmia, Bradycardia, Tachycardia, Management, Emergency, Ghana

Posted Date: November 19th, 2021

DOI: https://doi.org/10.21203/rs.3.rs-1056596/v1

License: (1) (1) This work is licensed under a Creative Commons Attribution 4.0 International License. Read Full License 


\section{Abstract}

\section{Background}

Management of life-threatening arrhythmia can be incredibly challenging in advanced health systems. In sub-Saharan Africa (SSA), this is likely more challenging because of weak pre-hospital and in-hospital critical care systems. Little is known about life-threatening arrhythmia, and their outcomes in SSA. The aim of this study was to examine the outcomes and types of arrhythmias among haemodynamically unstable patients presenting at a tertiary hospital in Accra-Ghana.

\section{Method}

This was a retrospective case series study conducted at the Korle-Bu Teaching Hospital (KBTH), AccraGhana. Medical records of patients who presented with or developed haemodynamically unstable arrhythmias within 24 hours of admission from January 2018 to December 2020 were reviewed. The demographic characteristics and clinical data including outcomes of patients were collected. Descriptive statistics was used, and results presented in frequency table.

\section{Results}

A total of 42 patients with life-threatening arrhythmias were included. Haemodynamically unstable tachyarrhythmias were the commonest arrhythmias found among the patients (70\%). Approximately, $52.5 \%$ of patients had structural heart diseases (52.5\%) whereas $27.5 \%$ had no apparent underlying cause or predisposing factor. Cardioversion (44.8\%), commonly electrical (31.0\%), and pacemaker implantation (34.5\%) were the common initial interventions. Majority of the patients (88.1\%) survived and were discharged home.

\section{Conclusion}

Tachyarrhythmias are the most common haemodynamically unstable arrhythmias seen among patients presenting emergently in a leading tertiary hospital in Ghana. The high survival rate observed was likely due to the presence of specialists on duty in our facility. This outcome cannot be extrapolated to other healthcare settings in sub-Saharan Africa with limited resources to manage these clinical entities.

\section{Background}

Arrhythmias refer to the rate, rhythm and conduction disturbances of the heart ${ }^{1}$, which may occur as bradyarrhythmia or tachyarrhythmia ${ }^{2}$. These disturbances could be caused by a range of underlying conditions including electrolyte abnormalities particularly hyperkalemia, endocrine disorders, structural heart diseases, respiratory conditions, drugs and post cardiac surgery ${ }^{1}$. Sudden onset of the arrhythmias could be an indication of systemic problems due to hypoxia, acid-base disturbances and multiple organ dysfunction ${ }^{3}$. Arrhythmias contribute significantly to hospital admissions, and are associated with high 
morbidity and mortality ${ }^{4}$. In Europe and North America, John et al noted that ventricular tachycardia (VT) or ventricular fibrillation (VF) accounts for almost $50 \%$ of sudden cardiac deaths ${ }^{5}$. Sustained VT and atrial fibrillation (AF) have been indicated to be the leading arrhythmias ${ }^{6}$. Arrhythmias are common conditions in critically ill patients ${ }^{7}$, and in emergency departments (ED), the most common arrhythmia is $A F^{8}$. A systematic review has documented a prevalence of $16-22 \%$ of Atrial fibrillation/flutter in heart failure and $3-7 \%$ in cardiology admissions in sub-Saharan Africa ${ }^{9}$.

The clinical presentation, 12-lead surface electrocardiogram and response to vagal manoeuvres or drugs are useful in diagnosing the underlying causes of the arrhythmia and in determining the appropriate management, particularly in the emergency settings ${ }^{10}$. However, there is, to a large extent, limited data on the emergency presentation of arrhythmias in sub-Saharan Africa ${ }^{9}$. The management and prognosis of patients depend on well-staffed emergency rooms and critical care units, both of which are poorly developed in sub-Saharan Africa ${ }^{11,12}$. We report on patients presenting with haemodynamically unstable arrhythmias at the Emergency Department (ED) of Korle-Bu Teaching Hospital (KBTH), Accra, Ghana.

\section{Methods}

\section{Study design and setting}

This study is a retrospective case series study carried out at the Korle-Bu Teaching Hospital (KBTH), Accra-Ghana. KBTH is the largest and leading national referral hospital in Ghana, with a bed capacity of 2,000 and about 1,500 out-patient department visits every day.

\section{Patient population}

Patients who presented with or developed haemodynamically unstable arrhythmias whilst on admission between January 2018 and December 2020 were recruited into the study. Haemodynamic instability was defined as blood pressure less than $90 / 60 \mathrm{mmHg}$ with associated signs of tissue hypoperfusion or shock. Patients' information included onset of admission until a clinical outcome. Clinical outcome was defined as either a discharge or death.

A structured extraction form was used to collect data on the age and sex, clinical presentations, underlying predisposing factor, therapy received and outcomes. Clinical examinations findings by physicians and the cardiologists at the hospital were also captured. Electrocardiograph (ECG) and echocardiogram were done for all the patients, and coronary angiogram was carried out among some of those suspected of having coronary heart disease. Arrhythmic events were diagnosed based on the clinical features and a confirmatory ECG finding. The ECGs were performed at either the referring hospitals or at the KBTH. ECG interpretations captured were those done by either physician specialists or cardiologists. The ECGs were also reviewed by the study team. Arrhythmia classification was done by 
analysing the ECG for arrhythmic events, and their occurrences were classified as either bradyarrhythmias or tachyarrhythmias.

Permission to use the clinical data was obtained from from the Ethical and Protocol Review Committee (EPRC) of the KBTH.

\section{Data analysis}

Data collected was entered into and analysed using Statistical Package for Social Sciences (SPSS) version 22.0. Categorical data was presented as frequencies and percentages. Continuous variables were presented as means and standard deviations.

\section{Results}

\section{Baseline characteristics}

A total of 42 patients aged 17 to 89 years (mean age $57.2 \pm 20.9$ ) were included in the study representing $0.114 \%$ or 114 in 100,000 of emergency medical admissions during the period. Majority of the patients were males (54.8\%). Pre-syncope (35.7\%), palpitations (28.6\%) and syncope (28.6\%) were the most common symptoms among patients (Table 1 ).

Table 1

Clinical presentations of the patients 


\begin{tabular}{|ll|}
\hline Characteristics & Frequency (\%) \\
\hline Age (years) & $57.2 \pm 20.9$ \\
\hline Male & \\
\hline Female & $23(54.8)$ \\
\hline Presentation* & $19(45.2)$ \\
\hline Palpitations & $12(28.6)$ \\
\hline Syncope & $12(28.6)$ \\
\hline Pre-syncope & $15(35.7)$ \\
\hline Chest pain & $6(14.3)$ \\
\hline Dyspnea & $6(14.3)$ \\
\hline Heart failure & $5(11.9)$ \\
\hline Loss of consciousness & $5(11.9)$ \\
\hline Cardiac arrest & $2(4.8)$ \\
\hline *One patient could have more than one presentation \\
\hline
\end{tabular}

\section{Arrhythmias among patients and their underlining pathologies}

Tachyarrhythmias were the most common type of arrhythmias observed among the patients (66.7\%). Common tachyarrhythmias observed included AF (21.4\%), supraventricular tachycardia (26.2\%), which were mainly atrioventricular re-entry tachycardia (AVRT) and atrioventricular nodal re-entry tachycardia (AVNRT), and VT (14.3\%). Heart blocks (28.6\%) of which two-thirds (66.6\%) was complete heart block, was the commonest bradyarrhythmia observed. One of the patients presented with brady-tachy syndrome or sick sinus syndrome (Table 2).

Table 2

Arrhythmia and structural observed among patients 


\begin{tabular}{|ll|}
\hline Arrhythmias & Frequency (\%) \\
\hline Bradyarrhythmia & $13(30.9)$ \\
\hline Tachyarrhythmias & $28(66.7)$ \\
\hline Tachy-brady (sick sinus) syndrome & $1(2.4)$ \\
\hline & \\
\hline Specific arrhythmias & \\
\hline Heart block & $12(28.6)$ \\
\hline Mobitz Il AV-Block & $2(17)$ \\
\hline Complete AV-Block & $10(83)$ \\
\hline Fast atrial fibrillation & $9(21.4)$ \\
\hline Atrial fibrillation with other arrhythmias & $5(55.6)$ \\
\hline Fast atrial flutter & $2(4.8)$ \\
\hline Supraventricular tachycardia (AVRT and AVNRT) & $11(26.2)$ \\
\hline Ventricular tachycardia & $6(14.3)$ \\
\hline Ventricular fibrillation & $3(7.1)$ \\
\hline Underlying pathologies & $11(26.2)$ \\
\hline Thyroid disease & $9(21.1)$ \\
\hline Structural heart disease & $1(2.4)$ \\
\hline Hypertensive heart disease & $22(52.4)$ \\
\hline Dilated cardiomyopathy & $9(21.4)$ \\
\hline Valvular heart disease & $4(9.5)$ \\
\hline Congenital heart disease (ASD) & $2(4.8)$ \\
\hline No identifiable cause & \\
\hline
\end{tabular}

Structural heart diseases (52.4\%) were found to be the major underlying pathology of arrhythmias among the patients. Hypertensive heart disease, ischaemic heart disease and dilated cardiomyopathy contributed to arrhythmias in $21.4 \%, 14.3 \%$ and $9.5 \%$ of the patients respectively. Other causes identified included Bundle of Kent (Wolff Parkinson White Syndrome), respiratory acidosis secondary to upper airway obstruction, iatrogenic from atenolol use, hyperkalaemia, pulmonary embolism, stroke, diabetic 
ketoacidosis and congenital long QT syndrome. However, approximately $26.2 \%$ of the patients had no known or identifiable causes of arrhythmias.

\section{Management of Arrhythmia}

Cardioversion (52.4\%), commonly electrical cardioversion (33.3\%), and pacemaker implantation (23.8\%) were the common acute therapies offered to terminate and treat the arrhythmias respectively. For long term therapy, anti-arrhythmic drugs (35.7\%)-commonly amiodarone (26.2\%), permanent pacemaker implantation (9.5\%) and percutaneous coronary intervention (7.1\%) were common therapies (Table 3).

\section{Table 3}

Therapies instituted for the management of Arrhythmia ( $n=42)$ 


\begin{tabular}{|ll|}
\hline Therapy & Frequency (\%) \\
\hline Spontaneous termination (without therapy) & $2(4.8)$ \\
\hline & \\
\hline Acute therapy & \\
\hline Valsalva Maneuver & $2(4.8)$ \\
\hline Cardioversion & $22(52.4)$ \\
\hline Chemical (antiarrhythmic drugs) & $8(19.0)$ \\
\hline Electrical & $14(33.3)$ \\
\hline Defibrillation & $3(4.8)$ \\
\hline Beta-blocker & $4(9.5)$ \\
\hline Pacemaker implantation & $10(23.8)$ \\
\hline CPR (for cardiac arrest) & $3(7.1))$ \\
\hline Hyperkalaemia correction & $3(7.1)$ \\
\hline & \\
\hline Long term therapy & $1(2.4)$ \\
\hline Antiarrhythmic drugs & $1(2.4)$ \\
\hline Amiodarone & $1(2.4)$ \\
\hline Flecainide & $15(5.7)$ \\
\hline Beta-blocker & $11(26.2)$ \\
\hline Mitral valve repair/replacement & $1(2.4)$ \\
\hline Pacemaker implantation & $3(7.1)$ \\
\hline Percutaneous coronary intervention & $1(2.4)$ \\
\hline ICD implantation & $1(9.1)$ \\
\hline ASD closure & \\
\hline Radiofrequency ablation & \\
\hline Heart failure management & \\
\hline Haemodialysis & \\
\hline
\end{tabular}


Majority of patients (88.1\%) were successfully treated and discharged. Five of the patients died, representing a mortality rate of $11.9 \%$. One died from repeated arrhythmias, two from cardiac arrest and one from a thyroid storm.

\section{Discussion}

In this study we report the presentation of life-threatening arrhythmias among patients at the ED of KBTH, Ghana. It was found that pre-syncope, palpitations and syncope were the common presenting complaints. Tachyarrhythmia was the prevalent arrhythmia. Arrhythmias were mostly caused by structural heart diseases and arrhythmias were terminated mostly using cardioversion in the acute phase and in the long-term with the use of anti-arrhythmic drugs.

Symptoms particularly syncope, palpitations and light-headedness caused by cardiac arrhythmias are indicated to be the common complaints among patients presenting at EDs and could be responsible for 3 to $4 \%$ of all ED visits ${ }^{11}$. This confirms the observation made in our study where syncope, pre-syncope and palpitations were the commonest presenting complaints. It has been estimated that approximately $90 \%$ of patients presenting with syncope of cardiac origin have ECG abnormalities ${ }^{12}$. Abbott stated that a clinical presentation of palpitations together with syncope, dizziness or near syncope most often suggests a life-threatening tachyarrhythmia ${ }^{13}$.

Our current study found tachyarrhythmias as the most common arrhythmia. This finding is in consonance with what has been found among critically ill patients. In Reinelt et al's study, out of 310 episodes of arrhythmias observed, 278(89.7\%) were tachyarrhythmias and 32(10.3\%) bradyarrhythmias $^{14}$. In contrast, an international meta-analysis reported bradyarrhythmias $(4.8 \%)$ as the most frequent life-threatening arrhythmias compared to tachyarrhythmias $(2.6 \%)$ among patients presenting at an ED with syncope ${ }^{15}$. The observed difference could be due to difference in the study designs used.

Heart blocks, AF, supraventricular tachycardia (AVRT and AVNRT) and VT were found as the most common haemodynamically unstable arrhythmias. AF has been indicated to be the commonest arrhythmia observed in $\mathrm{EDs}^{8}$. In a population-based study over an 8-year period, AF accounted for $0.5 \%$ of all ED visits ${ }^{16}$. This conforms, in part, to the finding of our study where AF was the most common tachyarrhythmia observed. In the current study about $56 \%$ of AF was observed together with other arrhythmias including atrial flutter, VT, VF and torsade de pointes. This was observed in five of the patients at different times during the admission.

Supraventricular tachycardia seen in our study is similar to the finding of the study by Brunetti et al ${ }^{17}$ which showed that junctional supraventricular arrhythmias (AVNRT) were the most common arrhythmias. Sustained VT is not common as it has been indicated to be responsible for only $0.05 \%$ of patients visiting the ED, however unsustained VT is a common finding with an incidence of 2 to $7 \%$ among patients in critical care settings ${ }^{18}$. 
In this current study, AV block was found to be the most frequent bradyarrythmia and the commonest arrhythmias seen in 12 patients (28.6\%); Mobitz II AV-block, and complete heart block. Significant AV conduction disturbances including second-degree type $2 \mathrm{AV}$-block, advanced AV-block, third-degree AVblock with junctional escape rhythm, ventricular escape rhythm have also been found among emergency medical service patients referred for syncope ${ }^{17}$.

One patient in the study had an episode of sick sinus or brady-tachy syndrome requiring permanent pacemaker implantation and beta-blocker therapy. A similar observation was also made among patients requiring a permanent pacemaker by van der Boon et $\mathrm{al}^{19}$.

The study found structural heart diseases as the most common underlying pathology for the arrhythmias. In a meta-analysis study, patients with left ventricular hypertrophy were found to be at higher odds of developing supraventricular arrhythmias ${ }^{20}$. Downey et al. has also reported of ischemic heart disease as more common in patients with arrhythmias ${ }^{21}$. In our study, congenital heart disease accounted for arrhythmia in one of the patients. In adults, it has been stated that arrhythmias are the frequent long-term complication of congenital heart disease ${ }^{22}$. In a study among patients with congenital heart disease, arrhythmias occurred in $32 \%$ of them after undergoing surgery ${ }^{23}$. Arrhythmia in one of the patients in our study was found to be caused by valvular heart disease particularly mitral valve prolapse. It has also been reported that mitral valve prolapse may cause ventricular arrhythmia and is an underestimated cause of arrhythmic sudden cardiac death particularly in young adults ${ }^{24}$. Dilated cardiomyopathy was found to cause arrhythmia in four (9.5\%) of the patients in our study. A study has also identified arrhythmias in $18.7 \%$ of patients with peripartum cardiomyopathy, a type of dilated cardiomyopathy ${ }^{25}$. However, $26.2 \%$ of the patients in the current study had no known underlying pathology for arrhythmias. This is comparable to findings established in patients with AF, the most frequent arrhythmia, where the cause of up to $30 \%$ of cases may not be known ${ }^{26}$.

On the management of arrhythmias, the study found that cardioversion mostly electric cardioversion as an acute therapy was carried out in 14 of the patients (33.3\%) to terminate arrhythmias on account of the hemodynamic instability. Cardioversion either chemical or electrical is employed to re-establish normal sinus rhythm to ease symptoms and restore sinus rhythm ${ }^{27}$ In the acute treatment of cardiac arrhythmias, it has been indicated that direct current transthoracic cardioversion is performed to reestablish normal sinus rhythm in haemodynamically unstable patients ${ }^{27}$. This could justify the observation made in our study. It was observed in the study that three of the patients were defibrillated, with one of them as many as 53 times. Hood et al have stipulated that repeating unsuccessful cardioversion with amplified power, varying the vector, use of paddles with manual compression, or a few permutations is frequently successful ${ }^{27}$. However, it has been recommended particularly in the acute treatment of AF that electrical cardioversion may be done in ED with 150-200 biphasic Joules as first energy setting in order to avoid repeated shocks and VF that could arise from uncoordinated cardioversion of AF with lower voltages ${ }^{8}$. However, in our study one patient with AF was electrically 
cardioverted with 90 Joules whereas in 2 of the patients with VF electric cardioversion with 120 joules with primary success.

Pacemaker was the second common acute therapy performed in our study, primarily for bradyarrhythmias. In patients presenting with hemodynamically unstable bradycardia, pacing the heart either transvenously or transthoracically is recommended ${ }^{27}$. This is in line with what was seen in our study.

\section{Conclusion}

The incidence of haemodynamically unstable acute arrhythmias at the ED of the KBTH has a case fatality rate of $11.9 \%$. Pre-syncope, palpitations and syncope were the common presenting complaints of patients presenting with haemodynamically unstable arrhythmias. Tachyarrhythmia was the most common arrhythmia among patients. Arrhythmias were mostly caused by structural heart diseases however in a significant number of patients identifying an underlying cause or predisposing factor was elusive. Patients were managed mostly by cardioversion or pacemaker implantation in the acute phase and on long term using anti-arrhythmic medication. Interventions should be readily made available in all referral hospitals for the successful management of emergency arrhythmias.

\section{Abbreviations}

- AF Atrial fibrillation

- AV Atrioventricular

- AVRT Atrioventricular re-entry tachycardia

- AVNRT Atrioventricular re-entry tachycardia

- ASD Atrial Septal Defect

- ECG Electrocardiograph

- ED Emergency department

- ICD Implantable Cardiac Defibrillator

- KBTH Korle-Bu Teaching Hospital

- VT Ventricular tachycardia

- VF Ventricular fibrillation

\section{Declarations}

\section{Conflict of interest}

The authors report of no conflict of interest in this research. 


\section{Authors Contributions}

AD: Conceptualized and design the study, reviewed, and approved the final version of the manuscript. BYAA: Analysed the data and drafted the initial version of the manuscript. RO: collected the data, assisted in data analysis, reviewed and approved the final version. CO: assisted in the design of the study and data analysis, reviewed and approved the final manuscript. RD reviewed the initial draft and approved the final version. JA: reviewed and approved the final version of the manuscript. EA reviewed the initial draft and approved the final version. DA: reviewed the final manuscript.

\section{Ethical Approval and Consent to Participate}

This study was conducted in accordance with internationally accepted scientific principles and code of ethics governing medical research as enshrined in the Helsinki declaration adopted by World Medical Association (2013) or The Nuremberg Code (1947).

Ethical approval was granted by the Ethical and Protocol Review Committee of the KBTH (Protocol Identification Number: KBTH-STC/IRB/000104/2021). Waiver for consent for the use of the clinical data was granted by the KBTH (dated $3^{\text {rd }}$ August 2021)

This was a consecutive retrospective case series involving the review of hospital data or patients records on the outcome of the management of life-threatening arrhythmia. Patients were not followed up directly whilst on admission. Hence ethical approval was based on retrospective review of patients records from 2018 to 2020 but not prospective or follow-up of patients whilst on admission. Hence, consent was not required by the ethical committee.

\section{Consent for Publication}

Not Applicable

\section{Funding}

No funding was obtained by the authors for this research.

\section{Acknowledgement}

We express our appreciation to the authorities at the Korle-Bu Teaching Hospital for their support and to the participants of this important study.

\section{Availability of Data and Material}


The datasets during and/or analysed during the current study available from the corresponding author upon reasonable request.

\section{References}

1. Ministry of Health (MOH). Standard Treatment Guidelines: Arrhythmias. 2017. Seventh Edition. Accra, Ghana. GNDP. 2017; pg156.

2. Chow G V., Marine JE, Fleg JL. Epidemiology of Arrhythmias and Conduction Disorders in Older Adults. Clin Geriatr Med. 2012;28(4):539-553. doi:10.1016/J.CGER.2012.07.003

3. Goodman S, Weiss Y, Weissman C. Update on cardiac arrhythmias in the ICU. Curr Opin Crit Care 2008;14:549-554. DOI: 10.1097/MCC.0b013e32830a4c5d.

4. Kumar S, Tedrow UB, Triedman JK. Arrhythmias in Adult Congenital Heart Disease: Diagnosis and Management. Cardiol Clin. 2015;33(4):571-588. doi:10.1016/J.CCL.2015.07.007

5. John R, Tedrow U, Koplan B, et al. Cariac Arrhythmia 3: Ventricular arrhythmias and sudden cardiac death. Lancet. 2012;380:1520-1529. doi:10.1016/S0140-6736(12)61413

6. Trappe HJ, Brandts B, Weismueller P. Arrhythmias in the intensive care patient. Current Opinion in Critical Care 2003; 9:345-355

7. Wong A, Pierce T. Cardiac arrhythmias in the critically ill. Anaesth Intensive Care Med. 2012;13(8):360-368. doi:10.1016/J.MPAIC.2012.05.006

8. Stiell IG, Macle L. Canadian Cardiovascular Society Atrial Fibrillation Guidelines 2010: Management of Recent-Onset Atrial Fibrillation and Flutter in the Emergency Department. Can J Cardiol. 2011;27(1):38-46. doi:10.1016/J.CJCA.2010.11.014

9. Yuyun MF, Bonny A, Ng GA, et al. A Systematic Review of the Spectrum of Cardiac Arrhythmias in Sub-Saharan Africa. Glob Heart. 2020;15(1):37. doi:10.5334/GH.808

10. Trappe H-J. Treating critical supraventricular and ventricular arrhythmias. J Emergencies, Trauma Shock. 2010;3(2):143. doi:10.4103/0974-2700.62114

11. Schreiber D, Sattar A, Drigalla D, Higgins S. Ambulatory Cardiac Monitoring for Discharged Emergency Department Patients with Possible Cardiac Arrhythmias. West J Emerg Med. 2014;15(2):194. doi:10.5811/WESTJEM.2013.11.18973

12. Sarasin FP, Louis-Simonet M, Carballo D, et al. Prospective evaluation of patients with syncope: a population-based study. Am J Med. 2001;111(3):177-184. doi:10.1016/S0002-9343(01)00797-5

13. Abbott A V. Diagnostic Approach to Palpitations. Am Fam Physician. 2005;71(4):743-750.

14. Reinelt P, Karth G, Geppert A, Heinz G. Incidence and type of cardiac arrhythmias in critically ill patients: a single center experience in a medical-cardiological ICU. Intensive Care Med 2001279. 2014;27(9):1466-1473. doi:10.1007/S001340101043

15. D'Ascenzo F, Biondi-Zoccai G, Reed MJ, et al. Gabayan GZ, Suzuki M, Costantino G, Furlan R, Rosso AD, Sarasin FP, Sun BC, Modena MG, Gaita F. Incidence, etiology and predictors of adverse outcomes in 43,315 patients presenting to the Emergency Department with syncope: An international meta- 
analysis. International Journal of Cardiology. 2011; 167 (2013):57-62.

doi:10.1016/j.ijcard.2011.11.083.

16. Atzema CL, Austin PC, Miller E, Chong AS, Yun L, Dorian P. A Population-Based Description of Atrial Fibrillation in the Emergency Department, 2002 to 2010. Ann Emerg Med. 2013;62(6):570-577.e7. doi:10.1016/J.ANNEMERGMED.2013.06.005

17. Brunetti ND, De Gennaro L, Dellegrottaglie G, Antonelli G, Amoruso D, Di Biase M. Prevalence of cardiac arrhythmias in pre-hospital tele-cardiology electrocardiograms of emergency medical service patients referred for syncope. J Electrocardiol. 2012;45(6):727-732.

doi:10.1016/J.JELECTROCARD.2012.07.018

18. Dresen WF, Ferguson JD. Ventricular Arrhythmias. Cardiol Clin. 2018;36(1):129-139. doi:10.1016/J.CCL.2017.08.007

19. van der Boon RMA, Van Mieghem NM, Theuns DA, Nuis R-J, Nauta ST, Serruvs PW, Jordens L, van Domburg RT, de Jaegere PPT. et al. Pacemaker dependency after transcatheter aortic valve implantation with the self-expanding Medtronic Core Valve System. International Journal of Cardiology 2013;168(2):1269-1273. DOI: https://doi.org/10.1016/j.ijcard.2012.11.115.

20. Chatterjee S, Bavishi C, Sardar P, et al. Meta-Analysis of Left Ventricular Hypertrophy and Sustained Arrhythmias. Am J Cardiol. 2014;114(7):1049-1052. doi:10.1016/J.AMJCARD.2014.07.015

21. Downey AW, Quach JL, Haase M, Haase-Fielitz A, Jones D, Bellomo R. Characteristics and outcomes of patients receiving a medical emergency team review for acute change in conscious state or arrhythmias. Crit Care Med. 2008;36(2):477-481. doi:10.1097/01.CCM.0000300277.41113.46

22. Ávila P, Mercier L-A, Dore A, et al. Adult Congenital Heart Disease: A Growing Epidemic. Can J Cardiol. 2014;30(12):S410-S419. doi:10.1016/J.CJCA.2014.07.749

23. Koyak Z, Achterbergh RCA, Groot JR de, et al. Postoperative arrhythmias in adults with congenital heart disease: Incidence and risk factors. Int J Cardiol. 2013;169(2):139-144. doi:10.1016/J.IJCARD.2013.08.087

24. Basso C, Marra MP, Rizzo S, et al. Arrhythmic Mitral Valve Prolapse and Sudden Cardiac Death. Circulation. 2015;132(7):556-566. doi:10.1161/CIRCULATIONAHA.115.016291

25. Mallikethi-Reddy S, Akintoye E, Trehan N, et al. Burden of arrhythmias in peripartum cardiomyopathy: Analysis of 9841 hospitalizations. Int J Cardiol. 2017;235:114-117. doi:10.1016/J.IJCARD.2017.02.084

26. Wyse DG, Van Gelder IC, Ellinor PT, et al. Lone Atrial Fibrillation: Does it Exist? J Am Coll Cardiol. 2014;63(17):1715-1723. doi:10.1016/J.JACC.2014.01.023

27. Hood RE, Shorofsky SR. Management of Arrhythmias in the Emergency Department. Cardiol Clin. 2006;24:125-133. doi:10.1016/j.ccl.2005.09.005 